\title{
Ring kinetic theory for an idealized granular gas
}

\author{
T.P.C. van Noije and M.H. Ernst \\ Instituut voor Theoretische Fysica, Universiteit Utrecht, Postbus 80006, 3508 TA Utrecht, \\ The Netherlands
}

(February 1, 2008)

\begin{abstract}
The dynamics of inelastic hard spheres is described in terms of the binary collision expansion, yielding the corresponding pseudo-Liouville equation and BBGKY hierarchy for the reduced distribution functions. Based on cluster expansion techniques we derive the Boltzmann and ring kinetic equations for inelastic hard spheres. In the simple ring approximation, we calculate the structure factor of vorticity fluctuations in a freely evolving, dilute granular gas. The kinetic theory result agrees with the result, derived previously from fluctuating hydrodynamics. In the limit of incompressible flow, this structure factor alone determines the spatial velocity correlations, which are of dynamic

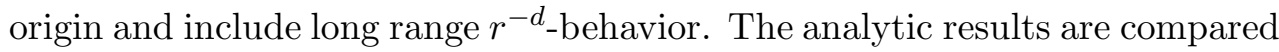
with MD simulations.
\end{abstract}

\section{INTRODUCTION}

In recent years the interest in static and rheological properties of assemblies of mesoscopic or macroscopic bodies or granules has been rapidly increasing. Both as a solid and as a fluid, granular systems show unusual behavior [1]. Flows of granular material can be subdivided into quasi static (contact) flows and rapid (collision driven) granular flows. In this characterization of Ref. [1], the former is referred to as the granular liquid regime and 
the latter as the granular gas regime. Only in rapid granular flows, the dynamics can be described by sequences of binary collisions and, as a consequence, the methods of kinetic theory are most suitable [2 [1]. Such flows do obey the standard conservation laws of mass and momentum, and can therefore be considered as fluids. However, energy is not conserved. The dynamics is essentially dissipative, which gives rise to several interesting new phenomena, such as clustering and inelastic collapse [1].

In rapid granular flows, collisions of granules are accompanied by conversion of kinetic energy into rotational energy and into energy of other internal degrees of freedom, and lead to effective 'cooling' phenomena in unforced flows. Such flows have been extensively modeled through smooth and rough hard spheres with inelastic collisions [2 [1].

Up to now the theoretical description of rapid granular flows has been based on Boltzmann-Enskog kinetic equations for hard sphere-type interactions. Inherent to such descriptions is the molecular chaos assumption of uncorrelated binary collisions. To study the extent to which collective effects may be of importance at a fundamental level of description, one needs to correct for the breakdown of the molecular chaos assumption and to account for the effects of dynamic correlations.

In the last 35 years many-body theories have been developed to account for these dynamic correlations in systems of microscopic particles obeying the standard conservation laws. The fundamental concept to describe these dynamic correlations are 'ring collisions', i.e. sequences of correlated binary collisions, which lead to the so called ring kinetic theory. This ring kinetic theory for systems of perfectly smooth elastic hard spheres has been at the basis of all major developments in nonequilibrium statistical mechanics over the last three decennia: it explains the logarithmic density dependence of the transport coefficients, and the breakdown of the virial expansion for transport coefficients [12 [14]; it explains the algebraic long time tails of the velocity autocorrelation function and similar current-current correlation functions [15 [17, the non-analytic dispersion relations for sound propagation and for relaxation of hydrodynamic excitations [18], as well as the breakdown of the NavierStokes equations in two-dimensional fluids at very long times and the non-existence of linear 
transport coefficients in 2-D [19]; moreover, it explains the existence of long range spatial correlations in nonequilibrium stationary states [20,21], driven by reservoirs which impose shear rates or temperature gradients, or in driven diffusive systems. Such systems violate the conditions of detailed balance and the stationary states are non-Gibbsian states [22].

The goal of this paper is to explain the long range correlations (in fact, intermediate range, as the algebraic tails are exponentially cut off at the largest scales), observed in molecular dynamics simulations of unforced flows of two-dimensional granular gases [11,23,24]. This will be done by extending the mean field-type Boltzmann-Enskog equation for rapid granular flows by including ring collisions. To do so we choose the model of perfectly smooth, but inelastic hard disks or spheres $(d=2,3)$ of diameter $\sigma$. The model incorporates the most fundamental feature of the dissipative dynamics of granular flow, namely the conversion of kinetic energy into internal energy. Moreover, it is a many-body system with well-defined and relatively simple dynamics, which has already widely been used in molecular dynamics simulations [7, [1],23,24].

The interactions between smooth inelastic hard spheres (IHS) only affect the translational degrees of freedom and are modeled by instantaneous collisions as in the case of elastic hard spheres. During a collision momentum will be transferred along the line joining the centers of mass of the two colliding particles, indicated by the vector $\boldsymbol{\sigma}$ pointing from the center of particle 2 to that of particle 1, as illustrated in Fig. 1.

Upon collision, inelastic hard spheres of equal mass $m$ change their velocities according to the collision rules

$$
\begin{aligned}
& \mathbf{v}_{1}^{*}=\mathbf{v}_{1}-\frac{1}{2}(1+\alpha)\left(\mathbf{v}_{12} \cdot \hat{\boldsymbol{\sigma}}\right) \hat{\boldsymbol{\sigma}} \\
& \mathbf{v}_{2}^{*}=\mathbf{v}_{2}+\frac{1}{2}(1+\alpha)\left(\mathbf{v}_{12} \cdot \hat{\boldsymbol{\sigma}}\right) \hat{\boldsymbol{\sigma}},
\end{aligned}
$$

where $\hat{\boldsymbol{\sigma}}=\boldsymbol{\sigma} / \sigma$ denotes a unit vector, $\mathbf{v}_{12}=\mathbf{v}_{1}-\mathbf{v}_{2}$, and $\alpha$ is the coefficient of normal restitution. Whereas the total momentum of the two particles is conserved in a collision, the total energy loss is $\frac{1}{4} m \epsilon\left(\mathbf{v}_{12} \cdot \hat{\boldsymbol{\sigma}}\right)^{2}$, where the coefficient of inelasticity is $\epsilon=1-\alpha^{2}$.

The restituting (precollision) velocities $\left(\mathbf{v}_{1}^{* *}, \mathbf{v}_{2}^{* *}\right)$ giving rise to $\left(\mathbf{v}_{1}, \mathbf{v}_{2}\right)$, are found by 
inverting collision rule (1) and given by (see Fig. 1)

$$
\begin{aligned}
& \mathbf{v}_{1}^{* *}=\mathbf{v}_{1}-\frac{1}{2}\left(1+\alpha^{-1}\right)\left(\mathbf{v}_{12} \cdot \hat{\boldsymbol{\sigma}}\right) \hat{\boldsymbol{\sigma}} \\
& \mathbf{v}_{2}^{* *}=\mathbf{v}_{2}+\frac{1}{2}\left(1+\alpha^{-1}\right)\left(\mathbf{v}_{12} \cdot \hat{\boldsymbol{\sigma}}\right) \hat{\boldsymbol{\sigma}}
\end{aligned}
$$

Note that this inversion is not possible if $\alpha=0$, which is a trivial limit.

The kinetic theory for inelastic hard spheres will be developed in close analogy with that for elastic ones. The rather singular streaming operators $S_{t}(\Gamma)$, which generate the $\Gamma$-space trajectories of the $N$-hard sphere system, are reformulated (see section $\llbracket$ II) in terms of $T$ operators (binary collision operators), following the original derivation in Ref. [25]. This allows us to introduce a pseudo-Liouville equation for the $N$-particle $\Gamma$-space distribution function, and obtain the Bogoliubov, Born, Green, Kirkwood and Yvon (BBGKY) hierarchy for the reduced distribution functions. The Liouville equation or the BBGKY hierarchy forms the standard starting point for deriving kinetic equations, such as the Boltzmann equation and the ring kinetic equation. This program is carried out in section III. Subsequently, we use the ring kinetic equation for inelastic hard spheres to calculate the nonequilibrium pair distribution function in a freely evolving, dilute granular gas.

A particular solution of the Boltzmann equation for this system is the so called homogeneous cooling state (HCS), where the velocity field vanishes everywhere, and the density $n$ and temperature $T(t)$ are spatially homogeneous, while the temperature decays in time. Some basic features of this HCS-solution are summarized in section IV. They are necessary for an understanding of the remaining part of this paper, including its instability against spatial fluctuations in flow velocity and density. In section $\mathrm{Q}$ we will calculate the buildup of vorticity correlations using the ring kinetic equation. The resulting expressions for the spatial correlations $\left\langle u_{\alpha}\left(\mathbf{r}+\mathbf{r}^{\prime}, t\right) u_{\beta}\left(\mathbf{r}^{\prime}, t\right)\right\rangle$ in the flow field, including long range $r^{-d_{-}}$ behavior, are identical to the results from fluctuating hydrodynamics for incompressible velocity fluctuations [23] in the low density limit. The incompressibility assumption gives a valid description of the velocity correlations up to a length scale that diverges as $1 / \epsilon$, beyond which the $r^{-d}$-tail is cut off exponentially. Section $\square$ concludes with a comparison of the 
theoretical predictions with the results of molecular dynamics simulations of unforced flows in a two-dimensional gas of inelastic hard disks (see [7:8,24]).

\section{THE BINARY COLLISION EXPANSION}

Having defined the dynamics of the system, we now turn to the statistical mechanics. The ensemble average of a dynamical quantity $A(\Gamma)$ obeys the equality

$$
\int \mathrm{d} \Gamma \rho(\Gamma, 0) A(\Gamma(t))=\int \mathrm{d} \Gamma A(\Gamma) \rho(\Gamma, t),
$$

where $\Gamma=\left\{x_{1}, x_{2}, \ldots x_{N}\right\}$ with $x_{i}=\left\{\mathbf{r}_{i}, \mathbf{v}_{i}\right\}$ is a point in the $N$-particle phase space. On the left hand side the time dependence is assigned to the dynamical variable $A(\Gamma(t)) \equiv$ $S_{t}(\Gamma) A(\Gamma)$ with $S_{t}(\Gamma)$ the time evolution operator, and on the right hand side to the $N$ particle distribution function $\rho(\Gamma, t)$, which can be done by considering the terms in (3) as an inner product. The time evolution of the $N$-particle distribution function is then given by

$$
\rho(\Gamma, t)=S_{t}^{\dagger} \rho(\Gamma, 0)
$$

where $S_{t}^{\dagger}(\Gamma)$ is the adjoint of $S_{t}(\Gamma)$. For Hamiltonian dynamics $S_{t}^{\dagger}(\Gamma)=S_{-t}(\Gamma)$.

For particles with hard core interactions the dynamics is undefined for physically inaccessible configurations, where the particles are overlapping. Such configurations have a vanishing weight in Eq. (3). Since $S_{t}(\Gamma)$ only appears in the combination $\rho(\Gamma, 0) S_{t}(\Gamma)$ which vanishes for overlapping initial configurations, it suffices to consider $W_{N}(\Gamma) S_{t}(\Gamma)$, with $W_{N}(\Gamma)=\prod_{i<j} W\left(r_{i j}\right)$ where $W(r)$ is the overlap function:

$$
W(r)=\left\{\begin{array}{l}
0 \text { if } r<\sigma \text { (overlapping) } \\
1 \text { if } r>\sigma \text { (non-overlapping) }
\end{array}\right.
$$

However, the methods of many-body theory require formal perturbation expansions and subsequent resummations. To do so, the time evolution operator $S_{t}(\Gamma)$ needs to be defined for all configurations, including the unphysical overlapping configurations. A convenient 
representation, defined in all points in phase space, has been developed for elastic hard spheres in Ref. [25], and is based on the binary collision expansion of $S_{t}(\Gamma)$ in terms of binary collision operators $T(i j)$.

The binary collision operator may be defined in terms of two-body dynamics through the time displacement operator $S_{t}(12)$, given in Ref. [25] by

$$
S_{t}(12)=S_{t}^{0}(12)+\int_{0}^{t} \mathrm{~d} \tau S_{\tau}^{0}(12) T(12) S_{t-\tau}^{0}(12) .
$$

Here the free streaming operator $S_{t}^{0}(12)=\exp \left[t L^{0}(12)\right]$, with $L^{0}(12)=L_{1}^{0}+L_{2}^{0}$ and $L_{i}^{0}=$ $\mathbf{v}_{i} \cdot \partial / \partial \mathbf{r}_{i}$. Its action on an arbitrary dynamical function is

$$
S_{t}^{0}(12) A\left(\mathbf{r}_{1}, \mathbf{p}_{1}, \mathbf{r}_{2}, \mathbf{p}_{2}\right)=A\left(\mathbf{r}_{1}+\mathbf{v}_{1} t, \mathbf{p}_{1}, \mathbf{r}_{2}+\mathbf{v}_{2} t, \mathbf{p}_{2}\right) .
$$

Following the argument of Ref. [25] for the case of elastic hard spheres step by step, the binary collision operator $T(12)$ for inelastic hard spheres is constructed as

$$
T(12)=\sigma^{d-1} \int_{\mathbf{v}_{12} \cdot \hat{\sigma}<0} \mathrm{~d} \hat{\boldsymbol{\sigma}}\left|\mathbf{v}_{12} \cdot \hat{\boldsymbol{\sigma}}\right| \delta\left(\mathbf{r}_{12}-\boldsymbol{\sigma}\right)\left(b_{\sigma}^{*}-1\right),
$$

where $b_{\sigma}^{*}$ is an operator that replaces all (precollision) velocities $\mathbf{v}_{i}(i=1,2)$ appearing to its right by postcollision velocities $\mathbf{v}_{i}^{*}$, defined for the inelastic case through collision rule (1), and $d$ is the dimensionality of the system.

The binary collision operator is defined for overlapping and non-overlapping configurations of two hard spheres. It extends the definition of $S_{t}(12)$ to all points in phase space. In the ensemble average considered in Eq. (3), the overlap function $W_{N}(\Gamma)$ contains a factor $W\left(r_{12}\right)$ which vanishes whenever $r_{12}<\sigma$. Moreover, the pseudo-dynamics introduced through Eq. (6) is noninvertible (see [25]). Consequently, the generator $S_{t}(12)$ for twoparticle dynamics is only defined for positive times. For later reference we quote the property $T(12) A(12)=0$, where $A(12)$ is a constant or a function of the argument $\left(\mathbf{v}_{1}+\mathbf{v}_{2}\right)$, because of conservation of particle number and linear momentum in binary collisions. As kinetic energy is not conserved in inelastic collisions, this property does not apply if $A(12)$ is a function of the argument $\left(v_{1}^{2}+v_{2}^{2}\right)$. 
Combinations of $T$-operators and free streaming operators $S_{t}^{0}$, preceded by appropriate combinations of overlap functions, can be used to construct the time displacement operators $S_{t}$ for dynamical variables. However to discuss the Liouville equation and describe the time evolution of the reduced distribution functions we need to consider the adjoint time displacement operators.

We start with the two-particle streaming operator $S_{t}(12)$. In order to obtain the adjoint $S_{t}^{\dagger}(12)$, we consider the integral equality

$$
\int \mathrm{d} x_{1} \mathrm{~d} x_{2} B(12) W(12) S_{t}(12) A(12)=\int \mathrm{d} x_{1} \mathrm{~d} x_{2} A(12) S_{t}^{\dagger}(12) W(12) B(12),
$$

where $\mathrm{d} x_{1} \mathrm{~d} x_{2}=\mathrm{d} \mathbf{r}_{1} \mathrm{~d} \mathbf{v}_{1} \mathrm{~d} \mathbf{r}_{2} \mathrm{~d} \mathbf{v}_{2}$ and $A(12)$ and $B(12)$ are arbitrary functions of the phases $x_{1}$ and $x_{2}$. Since $W(12)$ is appearing to the left of $S_{t}(12)$, this integral is well-defined. Substituting $S_{t}(12)$ from Eq. (6) and using Liouville's theorem $S_{-t}^{0 \dagger}(12)=S_{t}^{0}(12)$ for free particle motion, the left hand side of Eq. (9) can be written as

$$
\begin{aligned}
\int \mathrm{d} x_{1} \mathrm{~d} x_{2}\{ & A(12) S_{-t}^{0}(12) W(12) B(12)+ \\
& \left.\int_{0}^{t} \mathrm{~d} \tau\left[S_{-\tau}^{0}(12) W(12) B(12)\right] T(12) S_{t-\tau}^{0}(12) A(12)\right\} .
\end{aligned}
$$

Now notice that the binary collision operator $T(12)$ contains two terms: a real collision term with $b_{\sigma}^{*}$ and a virtual collision term. The real collision term appearing in (10) can be transformed using $\mathbf{v}_{12}^{*} \cdot \hat{\boldsymbol{\sigma}}=-\alpha \mathbf{v}_{12} \cdot \hat{\boldsymbol{\sigma}}$, yielding a Jacobian $\mathrm{d} \mathbf{v}_{1} \mathrm{~d} \mathbf{v}_{2}=\mathrm{d} \mathbf{v}_{1}^{*} \mathrm{~d} \mathbf{v}_{2}^{*} / \alpha$, and then relabeling $\mathbf{v}_{i}^{*} \rightarrow \mathbf{v}_{i}$ and $\mathbf{v}_{i} \rightarrow \mathbf{v}_{i}^{* *}$. In the virtual collision term we relabel $\hat{\boldsymbol{\sigma}} \rightarrow-\hat{\boldsymbol{\sigma}}$ and use the free particle Liouville theorem to finally write (10) in terms of the adjoint time displacement operator, defined through the right hand side of Eq. (9). The resulting expression for the adjoint time displacement operator becomes

$$
S_{t}^{\dagger}(12)=S_{-t}^{0}(12)+\int_{0}^{t} \mathrm{~d} \tau S_{-\tau}^{0}(12) \bar{T}(12) S_{-(t-\tau)}^{0}(12),
$$

where the binary collision operator $\bar{T}$ is defined as

$$
\bar{T}(12)=\sigma^{d-1} \int_{\mathbf{v}_{12} \cdot \hat{\sigma}>0} \mathrm{~d} \hat{\boldsymbol{\sigma}}\left(\mathbf{v}_{12} \cdot \hat{\boldsymbol{\sigma}}\right)\left(\frac{1}{\alpha^{2}} \delta\left(\mathbf{r}_{12}-\boldsymbol{\sigma}\right) b_{\sigma}^{* *}-\delta\left(\mathbf{r}_{12}+\boldsymbol{\sigma}\right)\right) .
$$


Here $b_{\sigma}^{* *}$ acts on the velocities $\mathbf{v}_{i}(i=1,2)$ to its right and replaces them by restituting ones, $\mathbf{v}_{i}^{* *}$, as defined in collision rule (2).

A property of $\bar{T}$-operators, equivalent to $T(12) A(12)=0$, is

$$
\int \mathrm{d} x_{1} \mathrm{~d} x_{2} A(12) \bar{T}(12) B(12)=0,
$$

if $A(12)$ is a constant or a function of the argument $\left(\mathbf{v}_{1}+\mathbf{v}_{2}\right)$, whereas $B(12)$ is an arbitrary function.

The time displacement operators $S_{t}(12)$ can be put in a more convenient form by using the property, $T(12) S_{t}^{0}(12) T(12)=0$, valid for any $t>0$. It also holds with $T$ replaced by $\bar{T}$. This relation expresses the fact that two hard spheres cannot collide more than once with only free propagation in between. Using this property, the time displacement operators can be written as

$$
\begin{array}{r}
W(12) S_{t}(12)=W(12) \exp \left[t L^{0}(12)+t T(12)\right] \\
S_{t}^{\dagger}(12) W(12)=\exp \left[-t L^{0}(1,2)+t \bar{T}(12)\right] W(12)
\end{array}
$$

We now return to the full $N$-particle system. As shown in Ref. [25], the dynamics of the $N$-particle system can be represented in the compact form of pseudo-streaming operators with the help of the above property $T(12) S_{t}^{0}(12) T(12)=0$, yielding

$$
\begin{array}{r}
W_{N}(\Gamma) S_{t}(\Gamma)=W_{N}(\Gamma) \exp \left[t L^{0}(\Gamma)+t \sum_{i<j} T(i j)\right] \\
S_{t}^{\dagger}(\Gamma) W_{N}(\Gamma)=\exp \left[-t L^{0}(\Gamma)+t \sum_{i<j} \bar{T}(i j)\right] W_{N}(\Gamma),
\end{array}
$$

with $L^{0}(\Gamma)=\sum_{i} L_{i}^{0}$ the free particle streaming operator. These time evolution operators are defined everywhere in phase space, and the overlap function gives a vanishing weight to unphysical configurations, provided that $W_{N}(\Gamma)$ always appears to the left of $T$-operators, or to the right of $\bar{T}$-operators.

A minor generalization is to include a conservative external force field in the dynamics. In that case the single-particle free streaming operator should be defined as

$$
L_{i}^{0}=\mathbf{v}_{i} \cdot \frac{\partial}{\partial \mathbf{r}_{i}}+\mathbf{a}_{i} \cdot \frac{\partial}{\partial \mathbf{v}_{i}}
$$


where $\mathbf{a}_{i}$ is the external force per unit mass, acting on the $i$-th particle.

Similar results for the binary collision operators $T$ and $\bar{T}$ for inelastic hard spheres have been derived independently by Brey et al. [5].

\section{BBGKY HIERARCHY}

The time evolution of the $N$-particle distribution function $\rho(\Gamma, t)=\rho\left(x_{1}, x_{2}, \ldots x_{N}, t\right)$, with $x_{i}=\left\{\mathbf{r}_{i}, \mathbf{v}_{i}\right\}$ is given by the Frobenius-Perron equation. For conservative Hamiltonian systems this equation is the Liouville equation which is an expression of the incompressibility of the flow. In the case of dissipative systems which by definition are time irreversible, the phase space volumes are contracted along the flow. According to Eqs. (4) and (15) the time evolution of the distribution function for inelastic hard spheres is given by the pseudoLiouville equation

$$
\left[\partial_{t}+L^{0}(\Gamma)\right] \rho(\Gamma, t)=\sum_{i<j} \bar{T}(i j) \rho(\Gamma, t)
$$

An equivalent representation of the time evolution of the system can be given in terms of reduced $s$-particle distribution functions $(s=1,2, \ldots)$, defined as

$$
f_{12 \ldots s}(t) \equiv f^{(s)}\left(x_{1}, x_{2}, \ldots x_{s}, t\right)=\frac{N !}{(N-s) !} \int \mathrm{d} x_{s} \ldots \mathrm{d} x_{N} \rho\left(x_{1}, x_{2}, \ldots x_{N}, t\right)
$$

where $\rho(t)$ is normalized to unity. Integration of Eq. (17) using (13) yields the BBGKY hierarchy for the reduced distribution functions. We only quote the first two hierarchy equations:

$$
\begin{aligned}
\left(\partial_{t}+L_{1}^{0}\right) f_{1} & =\int \mathrm{d} x_{2} \bar{T}(12) f_{12} \\
{\left[\partial_{t}+L_{1}^{0}+L_{2}^{0}-\bar{T}(12)\right] f_{12} } & =\int \mathrm{d} x_{3}[\bar{T}(13)+\bar{T}(23)] f_{123}
\end{aligned}
$$

This set of equations is an open hierarchy, which expresses the time evolution of the $s$-particle distribution function in terms of the $(s+1)$-th function.

In the literature on kinetic theory of inelastic hard spheres the first equation of the BBGKY hierarchy has frequently been derived intuitively and used as a starting point to 
obtain the Boltzmann-Enskog equation for the single-particle distribution function [2, 3, 5, 6]. Using the explicit expression (12) for $\bar{T}(12)$ it can be written in full detail as

$$
\begin{aligned}
& \left(\partial_{t}+L_{1}^{0}\right) f\left(\mathbf{r}_{1}, \mathbf{v}_{1}, t\right)=\sigma^{d-1} \int \mathrm{d} \mathbf{v}_{2} \int_{\mathbf{v}_{12} \cdot \hat{\sigma}>0} \mathrm{~d} \hat{\boldsymbol{\sigma}}\left(\mathbf{v}_{12} \cdot \hat{\boldsymbol{\sigma}}\right) \times \\
& \left\{\frac{1}{\alpha^{2}} f^{(2)}\left(\mathbf{r}_{1}, \mathbf{v}_{1}^{* *}, \mathbf{r}_{1}-\boldsymbol{\sigma}, \mathbf{v}_{2}^{* *}, t\right)-f^{(2)}\left(\mathbf{r}_{1}, \mathbf{v}_{1}, \mathbf{r}_{1}+\boldsymbol{\sigma}, \mathbf{v}_{2}, t\right)\right\} .
\end{aligned}
$$

The corresponding equation for the rate of change of an average is obtained by multiplying the first hierarchy equation in (19) with $\int \mathrm{d} \mathbf{v}_{1} \psi\left(\mathbf{v}_{1}\right)$, and using the adjoint of $\bar{T}(12)$ to find

$$
\begin{aligned}
& \frac{\partial}{\partial t} \int \mathrm{d} \mathbf{v}_{1} \psi\left(\mathbf{v}_{1}\right) f\left(\mathbf{r}_{1}, \mathbf{v}_{1}, t\right)+\frac{\partial}{\partial \mathbf{r}_{1}} \cdot \int \mathrm{d} \mathbf{v}_{1} \mathbf{v}_{1} \psi\left(\mathbf{v}_{1}\right) f\left(\mathbf{r}_{1}, \mathbf{v}_{1}, t\right) \\
= & \int \mathrm{d} \mathbf{v}_{1} \int \mathrm{d} x_{2} f_{12} T(12) \psi\left(\mathbf{v}_{1}\right) \\
= & \sigma^{d-1} \int \mathrm{d} \mathbf{v}_{1} \int \mathrm{d} \mathbf{v}_{2} \int_{\mathbf{v}_{12} \cdot \hat{\sigma}>0} \mathrm{~d} \hat{\boldsymbol{\sigma}}\left(\mathbf{v}_{12} \cdot \hat{\boldsymbol{\sigma}}\right) \times \\
& f^{(2)}\left(\mathbf{r}_{1}, \mathbf{v}_{1}, \mathbf{r}_{1}+\boldsymbol{\sigma}, \mathbf{v}_{2}, t\right)\left[\psi\left(\mathbf{v}_{1}^{*}\right)-\psi\left(\mathbf{v}_{1}\right)\right],
\end{aligned}
$$

where we have set the external force equal to zero. This equation is the starting point for deriving macroscopic conservation laws, hydrodynamic equations and the rate of change of the temperature.

We return again to the kinetic equations. In order to derive a closed equation for the single-particle distribution function $f$ or for the pair function $f_{12}$ some kind of closure relation is required, to express $f_{12}$ in terms of $f$, such as Boltzmann's molecular chaos assumption, Bogoliubov's functional assumption [26] or cluster expansion methods [14,27]. Here we shall illustrate how the methods derived in the kinetic theory for elastic hard spheres can be transferred directly to the case of inelastic hard spheres. This will be done by deriving the Boltzmann equation and the ring kinetic equation for the model under consideration.

The Boltzmann equation is obtained from the first hierarchy equation by keeping only terms to dominant order in the density. This implies the fundamental assumption of molecular chaos for dilute gases, expressing the absence of dynamic correlations between the velocities of two colliding particles just before collision, i.e. at $\left|\mathbf{r}_{12}\right|=\sigma+0$, 


$$
f_{12}=f\left(\mathbf{r}_{1}, \mathbf{v}_{1}, t\right) f\left(\mathbf{r}_{2}, \mathbf{v}_{2}, t\right) .
$$

Furthermore, in the low density limit the spatial separation between the colliding particles can be neglected, and the binary collision operator $\bar{T}(12)$, entering in the BBGKY hierarchy, reduces to

$$
\bar{T}(12)=\delta\left(\mathbf{r}_{12}\right) \bar{T}_{0}(12)=\delta\left(\mathbf{r}_{12}\right) \sigma^{d-1} \int_{\mathbf{v}_{12} \cdot \hat{\sigma}>0} \mathrm{~d} \hat{\boldsymbol{\sigma}}\left(\mathbf{v}_{12} \cdot \hat{\boldsymbol{\sigma}}\right)\left(\frac{1}{\alpha^{2}} b_{\sigma}^{* *}-1\right) .
$$

Then the nonlinear Boltzmann equation for inelastic hard spheres becomes

$$
\begin{aligned}
& \left(\partial_{t}+L_{1}^{0}\right) f\left(\mathbf{r}_{1}, \mathbf{v}_{1}, t\right)=\sigma^{d-1} \int \mathrm{d} \mathbf{v}_{2} \int_{\mathbf{v}_{12} \cdot \hat{\sigma}>0} \mathrm{~d} \hat{\boldsymbol{\sigma}}\left(\mathbf{v}_{12} \cdot \hat{\boldsymbol{\sigma}}\right) \times \\
& \left\{\frac{1}{\alpha^{2}} f\left(\mathbf{r}_{1}, \mathbf{v}_{1}^{* *}, t\right) f\left(\mathbf{r}_{1}, \mathbf{v}_{2}^{* *}, t\right)-f\left(\mathbf{r}_{1}, \mathbf{v}_{1}, t\right) f\left(\mathbf{r}_{1}, \mathbf{v}_{2}, t\right)\right\} \equiv I(f, f) .
\end{aligned}
$$

There are two significant differences with the Boltzmann equation for the elastic case: (i) the occurrence of $1 / \alpha^{2}$ in the gain term on the right hand side of (24); one factor $1 / \alpha$ comes from the Jacobian $\mathrm{d} \mathbf{v}_{1}^{* *} \mathrm{~d} \mathbf{v}_{2}^{* *}=(1 / \alpha) \mathrm{d} \mathbf{v}_{1} \mathrm{~d} \mathbf{v}_{2}$ and the other one from the reflection law $\mathbf{v}_{12}^{* *} \cdot \hat{\boldsymbol{\sigma}}=-(1 / \alpha) \mathbf{v}_{12} \cdot \hat{\boldsymbol{\sigma}}$ (see Fig. 1). (ii) In the inelastic case, the restituting precollision velocities, which yield $\left(\mathbf{v}_{1}, \mathbf{v}_{2}\right)$ as postcollision velocities, are different from the postcollision velocities $\left(\mathbf{v}_{1}^{*}, \mathbf{v}_{2}^{*}\right)$, which result from the direct precollision velocities. In the elastic case $(\alpha=1)$ the relation $\mathbf{v}_{i}^{*}=\mathbf{v}_{i}^{* *}$ holds.

The Boltzmann-Enskog equation for inelastic hard spheres (see Refs. [2, 3, 3, 6]) is obtained by replacing $f_{12}$ in the first hierarchy equation of (19) by $\chi\left(\mathbf{r}_{1}, \mathbf{r}_{2} \mid n\right) f_{1} f_{2}$, where $\chi\left(\mathbf{r}_{1}, \mathbf{r}_{2} \mid n\right)$ is the pair correlation function of elastic hard spheres in a spatially nonuniform equilibrium state (see Ref. [28]). This version of the molecular chaos assumption still neglects the velocity correlations, built up by sequences of correlated binary collisions, but does account for static short range correlations, caused by excluded volume effects. The consequence for the transport coefficients have been worked out in Refs. [2, 3],6].

As the density increases the contributions of correlated collision sequences to the collision term on the right hand side of (20) become more and more important. The most simple sequence of correlated collisions are the so called ring collisions; for example $(12)(13)(14) \ldots\left(23^{\prime}\right)\left(24^{\prime}\right) \ldots(12)$, ending with a recollision of the pair (12), which was 
involved in the first collision. In the intermediate time particle 1 collides, say, $s$ times with $s$ different particles $(3,4, \ldots)$, and particle 2 collides $s^{\prime}$ times with another set of $s^{\prime}$ different particles $\left(3^{\prime}, 4^{\prime}, \ldots\right)$. When particles 1 and 2 are about to recollide, they are dynamically correlated through their collision history, and the molecular chaos assumption (22) is no longer valid, i.e. $g_{12} \equiv f_{12}-f_{1} f_{2} \neq 0$.

A simple way to take these correlations into account at moderate densities has been given in Refs. 27,29. The method is based on a cluster expansion of the s-particle distribution functions, defined recursively as

$$
\begin{aligned}
f_{12} & =f_{1} f_{2}+g_{12} \\
f_{123} & =f_{1} f_{2} f_{3}+f_{1} g_{23}+f_{2} g_{13}+f_{3} g_{12}+g_{123},
\end{aligned}
$$

etc. Here $g_{12}$ accounts for pair correlations, $g_{123}$ for triplet correlations, etc. The molecular chaos assumption implies $g_{12}=0$, which is equivalent to (22). The basic assumption to obtain the ring kinetic equation is that the pair correlations are dominant and higher order ones can be neglected, i.e. $g_{123}=g_{1234}=\cdots=0$ in cluster expansion (25).

Substitution of (25) into ([19) and elimination of $\partial f_{i} / \partial t(i=1,2)$ from the second hierarchy equation using the first one, yields the ring kinetic theory of inelastic hard spheres:

$$
\begin{aligned}
& \left(\partial_{t}+L_{1}^{0}\right) f_{1}=\int \mathrm{d} x_{2} \bar{T}(12)\left(f_{1} f_{2}+g_{12}\right) \\
& {\left[\partial_{t}+L_{1}^{0}+L_{2}^{0}-\bar{T}(12)-\left(1+\mathcal{P}_{12}\right) \int \mathrm{d} x_{3} \bar{T}(13)\left(1+\mathcal{P}_{13}\right) f_{3}\right] g_{12}=\bar{T}(12)\left[f_{1} f_{2}+g_{12}\right] .}
\end{aligned}
$$

Here $\mathcal{P}_{i j}$ is a permutation operator that interchanges the particle labels $i$ and $j$. The second equation is the so called repeated ring equation for the pair correlation function. If the operator $\bar{T}(12)$ on the left hand side of the second equation is deleted, one obtains the simple ring approximation. Formally solving this equation for $g_{12}$ yields an expression in terms of the single-particle distribution functions $f_{i}(i=1,2,3)$, and subsequent substitution into the first hierarchy equation above yields the generalized Boltzmann equation in ring approximation. For a more detailed discussion of the collision sequences taken into account by Eq. (27) we refer to the original literature [27,29, 30]. 
The kinetic equations (26) and (27) constitute the new extensions of this article: the ring kinetic equations for inelastic hard spheres. All standard results for elastic hard spheres are recovered by setting the restitution coefficient $\alpha=1$. In this paper we will determine from (27) the behavior of the nonequilibrium pair distribution function $g\left(x_{1}, x_{2}, t\right)$ on hydrodynamic time scales, for a special solution $f(x, t)$ of the Boltzmann equation. The spatial correlations $\left\langle u_{\alpha}\left(\mathbf{r}+\mathbf{r}^{\prime}, t\right) u_{\beta}\left(\mathbf{r}^{\prime}, t\right)\right\rangle$ of the flow field $\mathbf{u}(\mathbf{r}, t)$, calculation of which is our direct goal in this paper, can be directly obtained from $g\left(x_{1}, x_{2}, t\right)$ by integration. In the next section will summarize some known results about the homogeneous cooling state which are necessary for an understanding of what follows later on.

\section{HOMOGENEOUS COOLING STATE}

A gas of elastic hard spheres will relax to local equilibrium on a (kinetic) time scale of a few mean free times, $t_{0} \sim l_{0} / v_{0}$, where $l_{0}$ is the mean free path and $v_{0}$ the thermal velocity, $\frac{1}{2} m v_{0}^{2} \equiv T$. Here the Boltzmann constant $k_{B}$ is set equal to unity. Finally, the system will reach a spatially homogeneous equilibrium state on a (hydrodynamic) time scale $\sim L / v_{0}$, where $L$ is the linear dimension of the system.

However, in the case of inelastic hard spheres kinetic energy is lost in collisions, and if the system is not driven, the kinetic energy associated with the thermal motion decreases, and interesting instabilities occur, such as clustering [7] and inelastic collapse [8].

Here we are interested in a special solution of the Boltzmann equation (24) for inelastic hard spheres, the so called homogeneous cooling state (HCS). In this state the distribution function $f(\mathbf{r}, \mathbf{v}, t)=f(\mathbf{v}, t)$, as well as the hydrodynamic fields are spatially homogeneous. These functions are defined as density $n(\mathbf{r}, t)=\int \mathrm{d} \mathbf{v} f(\mathbf{r}, \mathbf{v}, t)$, flow velocity $\mathbf{u}(\mathbf{r}, t)=$ $(1 / n(\mathbf{r}, t)) \int \mathrm{d} \mathbf{v} \mathbf{v} f(\mathbf{r}, \mathbf{v}, t)$, and granular temperature $T(\mathbf{r}, t)=(m / d n(\mathbf{r}, t)) \int \mathrm{d} \mathbf{v} V^{2} f(\mathbf{r}, \mathbf{v}, t)$, where $\mathbf{V}(\mathbf{r}, t)=\mathbf{v}-\mathbf{u}(\mathbf{r}, t)$ is the peculiar velocity. Furthermore, the flow velocity can be taken to vanish, $\mathbf{u}(\mathbf{r}, t)=\mathbf{0}$, and $n(\mathbf{r}, t)=n$ is constant in space and time. However,

$T(\mathbf{r}, t)=T(t)$ depends on time. Based on the fundamental concepts of the Chapman-Enskog 
theory, we expect that the single-particle distribution function $f$ after an initial transient of the order of a few mean free times, will only depend on time through its first few moments, which is here only the temperature $T(t)$. For dimensional reasons $f(\mathbf{v}, t)$ then takes the scaling form

$$
f(\mathbf{v}, t)=\frac{n}{v_{0}^{d}(t)} \tilde{f}\left(\frac{\mathbf{v}}{v_{0}(t)}\right)
$$

with the thermal velocity $v_{0}(t)$ depending on time. One can derive an integral equation for the unknown scaling form $\tilde{f}(\mathbf{c})$, with $\mathbf{c}=\mathbf{v} / v_{0}(t)$, by inserting (28) into the Boltzmann equation (24). The result is

$$
-\frac{1}{v_{0}^{2}} \frac{d v_{0}}{d t}\left(d+\mathbf{c}_{1} \cdot \frac{\mathrm{d}}{\mathrm{d} \mathbf{c}_{1}}\right) \tilde{f}\left(\mathbf{c}_{1}\right)=n \sigma^{d-1} \tilde{I}(\tilde{f}, \tilde{f})
$$

with

$$
\widetilde{I}(\tilde{f}, \tilde{f}) \equiv \int \mathrm{d} \mathbf{c}_{2} \int_{\mathbf{c}_{12} \cdot \hat{\sigma}>0} \mathrm{~d} \hat{\boldsymbol{\sigma}}\left(\mathbf{c}_{12} \cdot \hat{\boldsymbol{\sigma}}\right)\left(\frac{1}{\alpha^{2}} b_{\sigma}^{* *}-1\right) \tilde{f}\left(\mathbf{c}_{1}\right) \tilde{f}\left(\mathbf{c}_{2}\right) .
$$

To determine the rate of change of the temperature, we use Eq. (21) with $\psi(\mathbf{v})=v^{2}$ and $f_{12}=f\left(\mathbf{v}_{1}, t\right) f\left(\mathbf{v}_{2}, t\right)$, and calculate $v_{1}^{* 2}-v_{1}^{2}$. With the help of Eqs. (11) and (28), we obtain

$$
\frac{\mathrm{d} T}{\mathrm{~d} t}=-\frac{\Omega_{d}}{\sqrt{2 \pi}} m n \sigma^{d-1} v_{0}^{3} \gamma=-2 \omega \gamma T
$$

where $\omega$ is the time dependent collision frequency given in Boltzmann theory by $\omega=$ $\Omega_{d} n \sigma^{d-1} \sqrt{T / \pi m}$ with $\Omega_{d}=2 \pi^{d / 2} / \Gamma(d / 2)$ the surface area of a $d$-dimensional unit sphere, and $\gamma$ is a time independent cooling rate, defined by

$$
\begin{aligned}
\gamma & \equiv-\frac{\sqrt{2 \pi}}{d \Omega_{d}} \int \mathrm{d} \mathbf{c}_{1} c_{1}^{2} \widetilde{I}(\tilde{f}, \tilde{f}) \\
& =\left(\frac{\sqrt{2 \pi}}{d \Omega_{d}}\right) \frac{1-\alpha^{2}}{4} \int \mathrm{d} \mathbf{c}_{1} \int \mathrm{d} \mathbf{c}_{2} \int_{\mathbf{c}_{12} \cdot \hat{\sigma}>0} \mathrm{~d} \hat{\boldsymbol{\sigma}}\left(\mathbf{c}_{12} \cdot \hat{\boldsymbol{\sigma}}\right)^{3} \tilde{f}\left(\mathbf{c}_{1}\right) \tilde{f}\left(\mathbf{c}_{2}\right),
\end{aligned}
$$

which depends on the unknown scaling form $\tilde{f}(\mathbf{c})$.

At this stage, it is convenient to change to a new time variable $\tau$, defined through $\mathrm{d} \tau=\omega(T(t)) \mathrm{d} t$. Integration yields

$$
\tau=\frac{1}{\gamma} \ln \left(1+\gamma t / t_{0}\right)
$$


The time $\tau$ actually presents the average number of collisions suffered per particle. In the elastic limit $(\epsilon \rightarrow 0)$, it becomes the real time, $\tau=t / t_{0}$, measured in units of the mean free time $t_{0}=1 / \omega\left(T_{0}\right)$ at the initial temperature $T_{0}$.

Eq. (31) is readily integrated to obtain for the temperature

$$
T(t)=\frac{T_{0}}{\left(1+\gamma t / t_{0}\right)^{2}}=T_{0} \exp (-2 \gamma \tau)
$$

The above equation represents the well known algebraic decay law for the granular temperature in the homogeneous cooling statef (see Refs. [7, [8] and references therein).

Combining (29) and (31) yields an integral equation for the scaling form $\tilde{f}(\mathbf{c})$, i.e.

$$
\frac{\Omega_{d}}{\sqrt{2 \pi}} \gamma\left(d+\mathbf{c}_{1} \cdot \frac{\mathrm{d}}{\mathrm{d} \mathbf{c}_{1}}\right) \tilde{f}\left(\mathbf{c}_{1}\right)=\widetilde{I}(\tilde{f}, \tilde{f}),
$$

where $\gamma$ is given through (32) in terms of $\tilde{f}$ itself. By a moment expansion of this equation, it has been shown 31 that the fourth cumulant of $\tilde{f}$ is small for any value of the inelasticity $\epsilon$ both in $d=2$ and 3. This has been confirmed by a direct simulation Monte Carlo calculation of the fourth and sixth cumulant in the homogeneous cooling state of inelastic hard spheres $(d=3)$ [32. To a good approximation, therefore, the solution of (35) approaches a Maxwellian, i.e. $\tilde{f}(c) \approx \phi(c) \equiv \pi^{-d / 2} \exp \left(-c^{2}\right)$. Similarly, the dimensionless cooling rate (32) is well approximated by replacing $\tilde{f}(c)$ by $\phi(c)$ in (32):

$$
\gamma_{0}=-\frac{\sqrt{2 \pi}}{d \Omega_{d}} \int \mathrm{d} \mathbf{c}_{1} c_{1}^{2} \widetilde{I}(\phi, \phi)=\frac{1-\alpha^{2}}{2 d} .
$$

\section{RING KINETIC THEORY}

In this section we will derive the structure factor of transverse velocity or vorticity fluctuations, $S_{\perp}(k, t)$, for a freely evolving, dilute gas of inelastic hard spheres. In general

\footnotetext{
${ }^{1}$ Note that, because of the spatial homogeneity of $f$, this result is still valid in Enskog theory, for general densities, once the collision frequency has been increased by the factor $\chi(n)$, which is the local equilibrium pair correlation function at contact.
} 
velocity correlations can be described by an isotropic tensor $S_{\alpha \beta}(\mathbf{k}, t)$, related to the scalar isotropic functions $S_{\perp}(k, t)$ and $S_{\|}(k, t)$ via the decomposition [33]

$$
S_{\alpha \beta}(\mathbf{k}, t)=\hat{k}_{\alpha} \hat{k}_{\beta} S_{\|}(k, t)+\left(\delta_{\alpha \beta}-\hat{k}_{\alpha} \hat{k}_{\beta}\right) S_{\perp}(k, t),
$$

where hats denote unit vectors. The structure factors $S_{\perp}(k, t)$ and $S_{\|}(k, t)$ of transverse and longitudinal velocity fluctuations are also related to the energy spectrum function $E(k)$ in the theory of homogeneous turbulence [33].

The inverse Fourier transform of $S_{\alpha \beta}(\mathbf{k}, t)$ are the spatial correlations $G_{\alpha \beta}(\mathbf{r}, t)$. In terms of the microscopic velocity field $\mathbf{u}(\mathbf{r}, t)$, it may be defined as

$$
G_{\alpha \beta}(\mathbf{r}, t)=\frac{1}{V} \int \mathrm{d} \mathbf{r}^{\prime}\left\langle u_{\alpha}\left(\mathbf{r}+\mathbf{r}^{\prime}, t\right) u_{\beta}\left(\mathbf{r}^{\prime}, t\right)\right\rangle .
$$

A similar decomposition [33] holds for the spatial velocity correlations:

$$
G_{\alpha \beta}(\mathbf{r}, t)=\hat{r}_{\alpha} \hat{r}_{\beta} G_{\|}(r, t)+\left(\delta_{\alpha \beta}-\hat{r}_{\alpha} \hat{r}_{\beta}\right) G_{\perp}(r, t)
$$

Whereas the tensors $G_{\alpha \beta}(\mathbf{r}, t)$ and $S_{\alpha \beta}(\mathbf{k}, t)$ include self-correlations of particles, the correlation functions $g_{12}$ and $s_{12}$ (as defined below) occurring in the second hierarchy equation do not. Therefore, it is convenient to substract the self-correlation part and introduce the tensors $S_{\alpha \beta}^{+}(\mathbf{k}, t) \equiv S_{\alpha \beta}(\mathbf{k}, t)-T(t) \delta_{\alpha \beta} / n m$ and $G_{\alpha \beta}^{+}(\mathbf{r}, t) \equiv G_{\alpha \beta}(\mathbf{r}, t)-T(t) \delta_{\alpha \beta} \delta(\mathbf{r}) / n m$, which is regular at the origin and related to $g_{12}$ by

$$
G_{\alpha \beta}^{+}(\mathbf{r}, t)=\frac{1}{V} \int \mathrm{d} \mathbf{r}^{\prime} \frac{1}{n^{2}} \int \mathrm{d} \mathbf{v}_{1} \int \mathrm{d} \mathbf{v}_{2} v_{1 \alpha} v_{2 \beta} g\left(\mathbf{r}+\mathbf{r}^{\prime}, \mathbf{v}_{1}, \mathbf{r}^{\prime}, \mathbf{v}_{2}, t\right) .
$$

Incompressibility of velocity fluctuations then implies $S_{\|}^{+}(k, t)=0$, in which case $G_{\perp}^{+}(r, t)$ is related to $G_{\|}^{+}(r, t)$ by (see [33])

$$
G_{\perp}^{+}(r, t)=G_{\|}^{+}(r, t)+\frac{r}{d-1} \frac{\partial}{\partial r} G_{\|}^{+}(r, t) .
$$

As discussed in section [11], in the low density limit, $\bar{T}(12)=\delta\left(\mathbf{r}_{12}\right) \bar{T}_{0}(12)$, the first hierarchy equation reduces to the Boltzmann equation, and the second hierarchy equation to its simple ring approximation, 


$$
\left(\frac{\partial}{\partial t}+\mathbf{v}_{1} \cdot \frac{\partial}{\partial \mathbf{r}_{1}}+\Omega_{1}+\mathbf{v}_{2} \cdot \frac{\partial}{\partial \mathbf{r}_{2}}+\Omega_{2}\right) g_{12}=\delta\left(\mathbf{r}_{12}\right) \bar{T}_{0}(12) f_{1} f_{2},
$$

where $\Omega$ is minus the linearized Boltzmann collision operator, i.e. $(i=1,2)$

$$
\begin{aligned}
\Omega_{i} \psi\left(\mathbf{v}_{i}\right) \equiv & -\int \mathrm{d} x_{3} \bar{T}_{0}(i 3)\left(1+\mathcal{P}_{i 3}\right) f_{3} \psi\left(\mathbf{v}_{i}\right) \\
= & -\sigma^{d-1} \int \mathrm{d} \mathbf{v}_{3} \int_{\mathbf{v}_{i 3} \cdot \hat{\sigma}>0} \mathrm{~d} \hat{\boldsymbol{\sigma}}\left(\mathbf{v}_{i 3} \cdot \hat{\boldsymbol{\sigma}}\right)\left(\frac{1}{\alpha^{2}} b_{\sigma}^{* *}-1\right) \times \\
& \left\{f\left(\mathbf{v}_{i}, t\right) \psi\left(\mathbf{v}_{i}\right)+f\left(\mathbf{v}_{3}, t\right) \psi\left(\mathbf{v}_{3}\right)\right\} .
\end{aligned}
$$

The term on the right hand side of Eq. (42), which vanishes in the case of detailed balance, provides a source of correlations for inelastic hard spheres in the homogeneous cooling state. For a homogeneous distribution $f(\mathbf{v}, t), g\left(\mathbf{r}_{1}, \mathbf{v}_{1}, \mathbf{r}_{2}, \mathbf{v}_{2}, t\right)$ depends on $\mathbf{r}_{12}=\mathbf{r}_{1}-\mathbf{r}_{2}$ only, and the ring equation for the Fourier transform $s\left(\mathbf{k}, \mathbf{v}_{1}, \mathbf{v}_{2}, t\right) \equiv \int \mathrm{d} \mathbf{r}_{12} e^{-i \mathbf{k} \cdot \mathbf{r}_{12}} g\left(\mathbf{r}_{1}, \mathbf{v}_{1}, \mathbf{r}_{2}, \mathbf{v}_{2}, t\right)$ is given by

$$
\left(\partial_{t}+i \mathbf{k} \cdot \mathbf{v}_{12}+\Omega_{1}+\Omega_{2}\right) s_{12}=\bar{T}_{0}(12) f\left(\mathbf{v}_{1}, t\right) f\left(\mathbf{v}_{2}, t\right)
$$

Inspired by the scaling ansatz (28) for the homogeneous cooling solution of the Boltzmann equation, we write the density and temperature dependence of $s_{12}$ explicitly as

$$
s\left(\mathbf{k}, \mathbf{v}_{1}, \mathbf{v}_{2}, t\right)=\frac{n^{2}}{v_{0}^{2 d}(t)} \tilde{s}\left(\mathbf{k}, \mathbf{c}_{1}, \mathbf{c}_{2}, \tau\right)
$$

Again it is convenient to transform to the kinetic time $\tau$, so that Eq. (44) reduces to

$$
\left[\partial_{\tau}+\widetilde{\Lambda}_{12}(\mathbf{k})\right] \tilde{s}\left(\mathbf{k}, \mathbf{c}_{1}, \mathbf{c}_{2}, \tau\right)=l_{0} \sigma^{d-1} \widetilde{T}_{0}(12) \tilde{f}\left(\mathbf{c}_{1}\right) \tilde{f}\left(\mathbf{c}_{2}\right)
$$

with the mean free path $l_{0}=v_{0} / \omega$. Here we have introduced the notation $(j=1,2)$

$$
\begin{aligned}
\widetilde{\Lambda}_{j}(\mathbf{k}) & =i l_{0} \mathbf{k} \cdot \mathbf{c}_{j}+\gamma\left(d+\mathbf{c}_{j} \cdot \frac{\partial}{\partial \mathbf{c}_{j}}\right)+l_{0} n \sigma^{d-1} \tilde{\Omega}_{j} \\
\widetilde{\Lambda}_{12}(\mathbf{k}) & =\widetilde{\Lambda}_{1}(\mathbf{k})+\widetilde{\Lambda}_{2}(-\mathbf{k})
\end{aligned}
$$

defined in terms of the dimensionless quantities

$$
\begin{aligned}
\widetilde{\Omega} & \equiv \frac{1}{v_{0}(t) \sigma^{d-1} n} \Omega_{1} \\
\widetilde{T}_{0}(12) & =\frac{1}{v_{0}(t) \sigma^{d-1}} \bar{T}_{0}(12) .
\end{aligned}
$$


The formal solution for the case that all pair correlations vanish at the initial time $t=0$, is given by

$$
\begin{aligned}
\tilde{s}\left(\mathbf{k}, \mathbf{c}_{1}, \mathbf{c}_{2}, \tau\right)= & \frac{1-\exp \left[-\tau \widetilde{\Lambda}_{12}(\mathbf{k})\right]}{\widetilde{\Lambda}_{12}(\mathbf{k})} l_{0} \sigma^{d-1} \widetilde{T}_{0}(12) \tilde{f}\left(\mathbf{c}_{1}\right) \tilde{f}\left(\mathbf{c}_{2}\right) \\
= & \sum_{\lambda, \mu}-\frac{\exp \tau\left[z_{\lambda}(\mathbf{k})+z_{\mu}(\mathbf{k})\right]-1}{z_{\lambda}(\mathbf{k})+z_{\mu}(\mathbf{k})}\left|\psi_{\lambda}^{R}\left(\mathbf{c}_{1}, \mathbf{k}\right) \psi_{\mu}^{R}\left(\mathbf{c}_{2}, \mathbf{k}\right)\right\rangle \times \\
& \left\langle\psi_{\lambda}^{L}\left(\mathbf{c}_{1}, \mathbf{k}\right) \psi_{\mu}^{L}\left(\mathbf{c}_{2}, \mathbf{k}\right) \mid l_{0} \sigma^{d-1} \widetilde{T}_{0}(12) \tilde{f}\left(\mathbf{c}_{1}\right) \tilde{f}\left(\mathbf{c}_{2}\right)\right\rangle .
\end{aligned}
$$

In the second line we have made a decomposition in eigenfunctions of the operator $\widetilde{\Lambda}$ and used the bracket notation, with inner products representing integrals over $\mathbf{c}$. The left and right eigenfunctions satisfy the eigenvalue relations,

$$
\begin{aligned}
\widetilde{\Lambda}_{j}(\mathbf{k})\left|\psi^{R}\left(\mathbf{c}_{j}, \mathbf{k}\right)\right\rangle & =-z_{\lambda}(\mathbf{k})\left|\psi^{R}\left(\mathbf{c}_{j}, \mathbf{k}\right)\right\rangle \\
\left\langle\psi^{L}\left(\mathbf{c}_{j}, \mathbf{k}\right)\right| \widetilde{\Lambda}_{j}(\mathbf{k}) & =-z_{\lambda}(\mathbf{k})\left\langle\psi^{L}\left(\mathbf{c}_{j}, \mathbf{k}\right)\right| .
\end{aligned}
$$

The structure factor of transverse velocity fluctuations, $S_{\perp}^{+}(k, t)$, is then given by

$$
\begin{aligned}
S_{\perp}^{+}(k, t) & =\frac{1}{n^{2}} \int \mathrm{d} \mathbf{v}_{1} \int \mathrm{d} \mathbf{v}_{2} v_{1 \perp \alpha} v_{2 \perp \alpha} s\left(\mathbf{k}, \mathbf{v}_{1}, \mathbf{v}_{2}, t\right) \\
& =v_{0}^{2}(t)\left\langle c_{1 \perp \alpha} c_{2 \perp \alpha} \mid \tilde{s}\left(\mathbf{k}, \mathbf{c}_{1}, \mathbf{c}_{2}, \tau\right)\right\rangle .
\end{aligned}
$$

We state here without further derivation that the transverse velocity or shear modes are the only modes contributing to $S_{\perp}^{+}(k, t)$ (see also [11,23]). It can be shown that the eigenfunctions corresponding to the shear mode are $\psi_{\perp \alpha}=\mathbf{c} \cdot \hat{\mathbf{k}}_{\perp \alpha}=c_{\perp \alpha}$, where the subscript $\alpha$ denotes one of the $d-1$ degenerate shear modes. The dispersion relation for the relaxation rate $z_{\perp}(k)$ of the shear mode with wavenumber $k$ is $z_{\perp}(k)=\gamma_{0}\left(1-k^{2} \xi^{2}\right)$. The correlation length $\xi=\sqrt{\eta / \rho \omega \gamma_{0}}$ with $\eta \sim \sqrt{T(t)}$ the time dependent shear viscosity, $\rho=m n$ the mass density, $\omega \sim \sqrt{T(t)}$ the collision frequency and $\gamma_{0}=\epsilon / 2 d$. For small inelasticity $\xi$ diverges as $1 / \sqrt{\epsilon}$. So in calculating $S_{\perp}^{+}(k, t)$, we only have to calculate the quantity $\left\langle c_{1 \perp \alpha} c_{2 \perp \alpha} \mid l_{0} \sigma^{d-1} \widetilde{T}_{0}(12) \tilde{f}\left(\mathbf{c}_{1}\right) \tilde{f}\left(\mathbf{c}_{2}\right)\right\rangle=-\Omega_{d} l_{0} \sigma^{d-1} \gamma / \sqrt{2 \pi}=-\gamma / n \approx-\gamma_{0} / n$, as can easily be shown using Eq. (21). We then obtain for $S_{\perp}^{+}(k, t)$ the expression

$$
S_{\perp}^{+}(k, t)=\left(\frac{T(t)}{n m}\right) \frac{\exp \left[2 \gamma_{0} \tau\left(1-k^{2} \xi^{2}\right)\right]-1}{1-k^{2} \xi^{2}} .
$$


This low density result agrees with a previous result derived from fluctuating hydrodynamics [23], which, moreover, extends the validity of the above expression to higher densities.

The subsequent analysis of $G_{\perp}(r, t)$ and $G_{\|}(r, t)$, under the simplifying assumption that the fluctuating flow fields are incompressible, i.e. $S_{\|}^{+}(k, t)=0$, has been given in [23]. The full analysis for the compressible case can also be given, and yields only small modifications to the incompressible case, except at the largest scales, where the algebraic tails are cut off exponentially [31].

It is found that the spatial correlation functions $G_{\perp}(r, t)$ and $G_{\|}(r, t)$ show structure on spatial scales large compared to the mean free path $l_{0}$. In particular one obtains for asymptotically large $r$ the algebraic tails, $G_{\|}(r, t) \sim-(d-1) G_{\perp}(r, t) \sim A / r^{d}$ with explicit expressions for $A$ [31]. Furthermore, $G_{\|}(r, t)$ is positive everywhere, while $G_{\perp}(r, t)$ has a negative minimum, reflecting the presence of vortices in the system. The structure function $S_{\perp}^{+}(k, t)$ for the flow field in the inelastic hard sphere system is the analog of the energy spectrum function $E(k)$ in the theory of $2-D$ or $3-D$ homogeneous turbulence in incompressible fluids. The qualitative forms of $G_{\perp}(r, t)$ and $G_{\|}(r, t)$ are roughly similar to the shapes of these functions in the theory of homogeneous turbulence, as illustrated in Fig. 3.2 of chapter 3 in Ref. [33]. For a more detailed description of these functions, we refer to [31].

We now return to the structure factors, and compare Eq. (52) with results from a twodimensional molecular dynamics simulation of $N=50000$ inelastic hard disks at a low area fraction $\phi=\frac{1}{4} \pi n \sigma^{2}=0.05$, and a coefficient of normal restitution $\alpha=0.85$. Fig. 2a shows the prediction of Eq. (52) in the low density limit at $\tau=30$ collisions per particle, together with simulation results for $S_{\perp}(k, t)$ and $S_{\|}(k, t)$, as obtained by performing a Fourier transform on the momentum fields, coarse-grained into $256 \times 256$ boxes, followed by a circular average. At the corresponding time $t$, Eq. (33), gives the slightly smaller value $\tau=28.7$ for the number of collisions per particles. Already at this density, Enskog theory gives a quantitatively significant increase of the collision frequency by a factor $\chi \simeq 1.08$, leading to a predicted number of collisions per particle $\tau=29.7$, for the case under consideration. For a more detailed comparison of both $S_{\perp}(k, t)$ and $S_{\|}(k, t)$ at general densities, we refer to 31. 
Since the structure factor of transverse velocity fluctuations, $S_{\perp}^{+}(k, t)$, shows structure at wavenumbers $k \lesssim 1 / \xi, \simeq 0.06 \sigma^{-1}$ in the ring kinetic theory, the spatial velocity correlation functions $G_{\perp}(r, t)$ and $G_{\|}(r, t)$ (shown in Fig. 2b) also show structure up to and beyond distances of the order $2 \pi \xi \simeq 106 \sigma$, which is large compared to the Boltzmann mean free path $l_{0} \simeq 6.3 \sigma$, but still small compared to the system size $L=886 \sigma$. Furthermore, the simulation results show that the incompressibility assumption indeed holds in a range of wavenumbers $1 / \xi_{\|} \lesssim k<1 / l_{0}$, where the existence of a large distance cut-off length $2 \pi \xi_{\|} \gg 2 \pi \xi$ has been discussed in Ref. [31]. The spatial velocity correlations measured in the simulations agree well with the calculated $G_{\perp}(r, t)$ and $G_{\|}(r, t)$ in the range $2 \pi l_{0}<r \lesssim 2 \pi \xi_{\|}$, and exhibit an observable long range $r^{-d}$-tail for distances $2 \pi \xi \lesssim r \lesssim 2 \pi \xi_{\|}$. At wavenumbers of the order of the minimal accessible wavenumber $k_{\min }=2 \pi / L \simeq 0.007 \sigma^{-1}$, effects from the periodic boundaries become important. To conclude, we observe that the more fundamental ring kinetic theory yields results identical to the more mesoscopic theory of fluctuating hydrodynamics in their common region of validity, thus supporting the phenomenological theory presented in Ref. 23,31.

The authors want to thank R. Brito and J.A.G. Orza, who performed the molecular dynamics simulations, for fruitful collaboration, and H.J. Bussemaker and D. Montgomery for stimulating discussion. T.v.N. acknowledges support of the foundation 'Fundamenteel Onderzoek der Materie (FOM)', which is financially supported by the Dutch National Science Foundation (NWO). 


\section{REFERENCES}

[1] H.M. Jaeger, S.R. Nagel and R.P. Behringer, Rev. Mod. Phys. 68, 1259 (1996).

[2] C.K.K. Lun, S.B. Savage, D.J. Jeffrey and N. Chepurnity, J. Fluid Mech. 140, 223 (1984).

[3] J.T. Jenkins and M.W. Richman, Arch. Rat. Mech. Anal. 87, 355 (1985); Phys. Fluids 28, 3485 (1985); J. Fluid Mech. 192, 313 (1988).

[4] J.J. Brey, F. Moreno and J.W. Dufty, Phys. Rev. E 54, 445 (1996).

[5] J.J. Brey, J.W. Dufty and A. Santos, J. Stat. Phys., June issue (1997).

[6] A. Goldshtein and M. Shapiro, J. Fluid Mech. 282, 75 (1995).

[7] I. Goldhirsch and G. Zanetti, Phys. Rev. Lett. 701619 (1993); I. Goldhirsch, M-L. Tan and G. Zanetti, J. Scient. Comp. 8, 1 (1993); N. Sela, I. Goldhirsch and S.H. Noskowicz, Phys. Fluids 8, 2337 (1996).

[8] S. McNamara, Phys. Fluids A 5, 3056 (1993); S. McNamara and W.R. Young, Phys. Rev. E 50, R28 (1994); 53, 5089 (1996).

[9] P. Deltour and J.-L. Barrat, J. Phys. I France 7, 137 (1997).

[10] S.E. Esipov and T. Pöschel, J. Stat. Phys. 86, 1385 (1997).

[11] J.A.G. Orza, R. Brito, T.P.C. van Noije and M.H. Ernst, cond-mat/9702029.

[12] S.G. Brush, Kinetic Theory, Vol. 3 (Pergamon Press, Oxford 1972), p. 77.

[13] J.M.J. van Leeuwen and A. Weyland, Physica 36, 457 (1967); 39, 35 (1968).

[14] J.R. Dorfman and H. van Beijeren, The Kinetic Theory of Gases, in Statistical Mechanics, Part B: Time-Dependent Processes, ed. B.J. Berne (Plenum Press, New York, 1977), ch. 3.

[15] B.J. Alder and T.E. Wainwright, Phys. Rev. A 1, 18 (1970). 
[16] M.H. Ernst, E.H. Hauge and J.M.J. van Leeuwen, Phys. Rev. Lett. 25, 1254 (1970).

[17] J.R. Dorfman and E.G.D. Cohen, Phys. Rev. Lett. 25, 1257 (1970).

[18] M.H. Ernst, Physica D 47, 198 (1991).

[19] Y. Pomeau and P. Resibois, Phys. Rep. 19C, 63 (1975).

[20] J.R. Dorfman, T.R. Kirkpatrick and J.V. Sengers, Annu. Rev. Phys. Chem. 45, 213 (1994).

[21] B. Schmittmann and R.K.P. Zia, Statistical mechanics of driven diffusive systems (Academic Press, 1995).

[22] H.J. Bussemaker, M.H. Ernst and J.W. Dufty, J. Stat. Phys. 78, 1521 (1995); H.J. Bussemaker and M.H. Ernst, Phys. Rev. E 53, 5837 (1996).

[23] T.P.C. van Noije, M.H. Ernst, R. Brito and J.A.G. Orza, Mesoscopic Theory of Granular Fluids, submitted Febr. 1997.

[24] R. Brito, J.A.G. Orza, T.P.C. van Noije and M.H. Ernst, to be published.

[25] M.H. Ernst, J.R. Dorfman, W.R. Hoegy and J.M.J. van Leeuwen, Physica 45, 127 (1969).

[26] E.G.D. Cohen, in Fundamental Problems in Statistical Mechanics, Vol. 1, ed. E.G.D. Cohen (North Holland Publ. Co., Amsterdam, 1962), p. 110.

[27] M.H. Ernst and J.R. Dorfman, Physica 61, 157 (1972).

[28] H. van Beijeren and M.H. Ernst, Physica 68, 437 (1973); Phys. Lett. 43A, 367 (1973).

[29] J.R. Dorfman and E.G.D. Cohen, J. Math. Phys. 8, 282 (1967).

[30] J.V. Sengers, M.H. Ernst and D.T. Gillespie, J. Chem. Phys. 56, 5583 (1972).

[31] T.P.C. van Noije, R. Brito and M.H. Ernst, to be published. 
[32] J.J. Brey, M.J. Ruiz-Montero and D. Cubero, preprint (1996).

[33] G.K. Batchelor, The Theory of Homogeneous Turbulence (Cambridge University Press, 1970), ch. 3. 


\section{FIGURES}

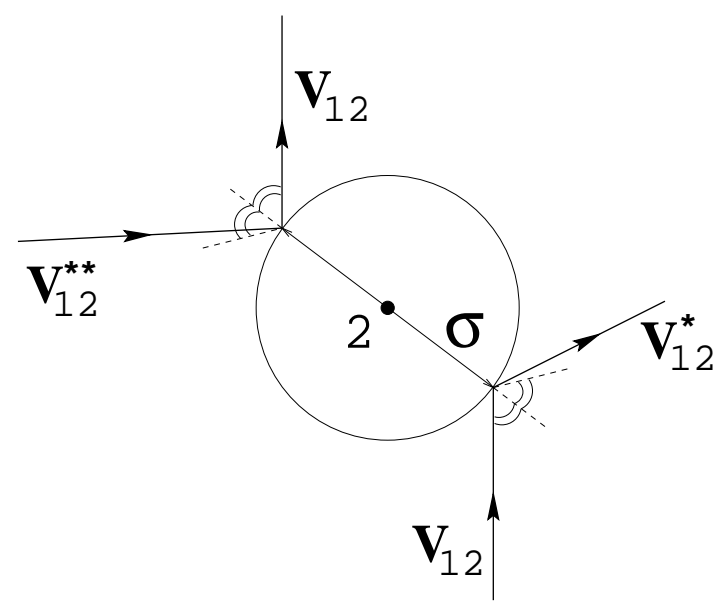

FIG. 1. Direct and restituting inelastic collisions with refection law $\mathbf{v}_{12}^{*} \cdot \hat{\boldsymbol{\sigma}}=-\alpha \mathbf{v}_{12} \cdot \hat{\boldsymbol{\sigma}}$ with $0<\alpha<1$.

(a)

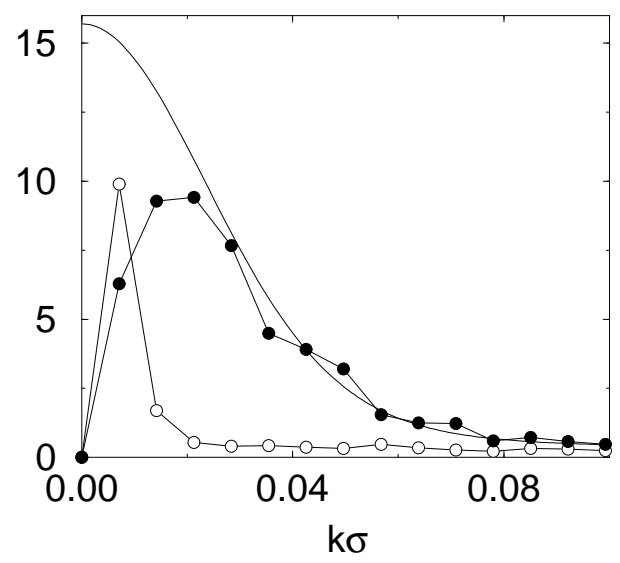

(b)

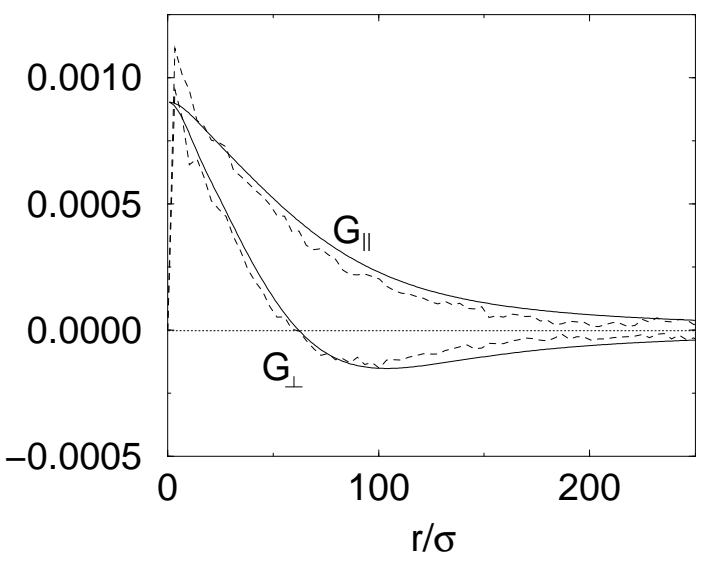

FIG. 2. (a) $S_{\perp}(k, t)$ (filled circles) and $S_{\|}(k, t)$ (open circles) as measured from molecular dynamics simulations of $N=50000$ particles at an area fraction $\phi=0.05$, coefficient of normal restitution $\alpha=0.85$ and number of collisions per particle $\tau=30$, with $T_{0} / m=1$, compared with the prediction Eq. (52) from ring kinetic theory for $S_{\perp}(k, t)$ (solid line). (b) Corresponding measured $G_{\|}(r, t)$ and $G_{\perp}(r, t)$ (dashed lines), compared with the prediction (solid lines) from ring kinetic theory in the incompressible limit. 